Revista de Investigación Educativa 25

julio-diciembre, 2017 | ISSN 1870-5308 | Xalapa, Veracruz

Instituto de Investigaciones en Educación | Universidad Veracruzana

\title{
Tutoría en Educación Superior: análisis desde diferentes corrientes psicológicas e implicaciones prácticas
}

\section{Tutoring in Higher Education: analysis from different approaches psychological and practical implications}

\author{
Dra. Gabriela de la Cruz Flores \\ Investigadora Asociada \\ Instituto de Investigaciones sobre la Universidad y la Educación, \\ Universidad Nacional Autónoma de México, México \\ gabydc@unam.mx
}

Los programas de tutoría en educación superior buscan abatir problemas centrales del sistema educativo como el abandono y el rezago escolar, aspectos que pueden caracterizarse como manifestaciones de la calidad y pertinencia social de la oferta educativa. En ese sentido, la tutoría puede emplearse a manera de un mecanismo que favorezca la equidad y la justicia educativa. La creación y funcionamiento de dichos programas se ha justificado primordialmente desde el plano de la política educativa y la normatividad; sin embargo, aún queda pendiente la discusión y el desarrollo de marcos teóricos y metodológicos específicos que articulen la acción tutorial en tanto una actividad educativa compensatoria. El presente artículo pretende contribuir a la construcción de marcos de referencia desde el campo de la psicología que contribuyan a conformar prácticas didácticas para la labor tutorial en educación superior. El análisis propuesto puede ser una guía para valorar las perspectivas imperantes y redefinir el qué y el cómo de los programas de tutoría orientados a promover la equidad educativa.

Palabras clave: Tutoría; tutores; educación superior; modelos teóricos. 
Mentoring programs in higher education seek to abate central problems of the educational system like abandonment and educational underachievement, both aspects are manifestations of quality and social relevance of educational provision, in that sense tutoring can be also a resource to promote equity and educational justice. The creation and operation of mentoring programs it has been justified primarily from the level of educational policy and regulatory aspects, however the discussion and development of specific theoretical and methodological frameworks that articulate the tutorial as a compensatory educational activity is pending. This article seek to contribute to the construction of theoretical and methodological mentoring frameworks, supported in the field of psychology, from which educational implications for higher education tutorial work arise. The proposed analysis can be a guide to assess the prevailing perspectives and redefine what and how mentoring programs could be transformed in a factor of educational equity.

Key words: Mentoring; tutors; higher education; theoretical models.

\title{
Tutoría en Educación Superior: análisis desde diferentes corrientes psicológicas e implicaciones prácticas
}

\author{
Tutoring in Higher Education: analysis from different \\ approaches psychological and practical implications
}

\section{Introducción}

a tutoría en educación superior ha cobrado un peso importante como una es-
trategia de intervención orientada a la atención del estudiantado a lo largo de su formación profesional. Como estrategia, la tutoría en general se ha enfocado en brindar herramientas que contribuyan al logro académico, con lo cual se ha aportado a la personalización de los procesos educativos. Cuando en torno a la tutoría se definen propósitos, estructuras, organización, apoyos, recursos, mecanismos de apoyo y sistemas de evaluación, se configuran programas de tutoría. En nuestro país los programas de tutoría en educación superior han tenido un crecimiento notable, 
impulsados por la Asociación Nacional de Universidades e Instituciones de Educación Superior (ANUIES) a través del documento eje titulado Programas Institucionales de Tutoría (2000). Actualmente la mayoría de las instituciones afiliadas a la ANUIES cuenta con un programa de tutoría. Desde su origen, los programas de tutoría surgieron como mecanismos para personalizar los procesos formativos y con ello contribuir a la disminución de problemas relacionados con el abandono y el rezago educativo. Dichos programas, además de canalizar y optimizar la gama de apoyos, servicios y recursos con los que cuentan las instituciones de educación superior para el fomento del aprendizaje y el desarrollo integral de los estudiantes, contemplan la intervención de la figura de los tutores, quienes en general son docentes y en algunos casos estudiantes (tutoría entre pares). Dentro de las actividades básicas de los tutores se identifican el seguimiento de las trayectorias formativas de los tutorados, en aras de ofrecer herramientas y estrategias que contribuyan a la mejora del aprendizaje y al rendimiento académico, así como la canalización de aquéllos a programas o servicios que la institución ofrezca a fin de atender necesidades específicas que quedan fuera del alcance del tutor (ANUIES, 2000).

Si bien se destaca la importancia de los programas de tutoría en beneficio del aprendizaje del estudiantado de educación superior y como una opción para personalizar procesos formativos masificados, dada la amplia matrícula de dicho nivel educativo, también habría que señalar que su incorporación y crecimiento han estado ligados a políticas educativas que pretenden elevar la calidad de las instituciones de educación superior (vía la eficiencia terminal y la titulación) y al reconocimiento de la actividad docente; tal es el caso del Programa para el Desarrollo Profesional Docente (PRODEP) que en relación al perfil deseable subraya:

El reconocimiento a Profesores/as de Tiempo Completo con perfil deseable se refiere al profesor/a universitario que posee un nivel de habilitación académica superior al de los programas educativos que imparte, preferentemente cuenta con el doctorado y, además, realiza de forma equilibrada actividades de docencia, generación o aplicación innovadora de conocimientos, tutorías y gestión académicavinculación. (Secretaría de Gobernación, p. 17)

En el plano internacional, los programas de tutoría en educación superior han cobrado relevancia; tal es el caso del Espacio Europeo donde, según Cano (2008), la convergencia entre países para consolidar el Espacio Europeo de Educación Superior (convocado en la Declaración de la Sorbona en 1998 y concretado en los Acuerdos de Bolonia en 1999) incluyó, entre otras cosas, recuperar a la tutoría como una vía para 
mejorar la calidad de la enseñanza universitaria. En palabras del autor referido, la tutoría coadyuva a:

transformar profundamente los procedimientos centrados en el aprendizaje de los estudiantes de cara a proporcionarles, a lo largo de su paso por la institución, una formación tan completa como sea posible y, por supuesto, favorecer su inserción profesional en el mundo laboral desde la plataforma de un Modelo Organizativo de Orientación Universitaria y de Acción Tutorial formalmente institucional. La Tutoría, pues, aparece así como uno de los mimbres a tener en cuenta en el tejido de la nueva Universidad; como un elemento inherente y consustancial a la función docente, como un factor de concreción práctica de la actividad orientadora de carácter integral; en definitiva, como un elemento de calidad y excelencia de la Educación Superior. (Cano, 2008, pp. 187-188)

La relevancia de la tutoría en la educación superior contemporánea también se justifica cuando se analiza la heterogeneidad de los perfiles de los estudiantes (amplia gama de edades, orígenes sociales, formación, habilidades e incluso expectativas sobre la educación superior); por ello la tutoría puede ser un mecanismo útil para personalizar y diversificar los procesos de aprendizaje. Por otra parte, la sociedad del conocimiento demanda a la formación profesional contender continuamente con la incertidumbre y la vorágine de la información, por lo que es imprescindible la generación de procesos de autorregulación en el estudiantado. Dicha formación no sólo implica el dominio de conocimientos disciplinarios específicos, sino el desarrollo de capacidades que integren habilidades de aprendizaje a lo largo de la vida. Lo anterior requiere de mecanismos formativos personalizados como los derivados del quehacer tutorial, los cuales pueden orientarse al desarrollo de proyectos personales y profesionales que les permitan a los estudiantes desempeñarse con ética y eficacia en los múltiples contextos de la actividad profesional. Al respecto, en un número monográfico destinado al análisis de la tutoría y sistemas de orientación en educación superior, Lobato e Ilvento (2013) refieren que el campo de la orientación y la tutoría se ha reformulado, a fin de coadyuvar en la construcción de proyectos de vida del estudiantado que promuevan su desarrollo integral y el acompañamiento en sus procesos de reflexión y de resolución de problemas, tanto en el plano personal como profesional y laboral. Para dichos autores esta reformulación enfatiza dos aspectos: el compromiso de la persona en la construcción de su propio proyecto de vida (incluyendo el ámbito profesional) y el necesario acompañamiento por profesionales en diferentes modalidades y contextos. Es en este último aspecto donde la figura del tutor cobra relevancia. 
El estudio de la tutoría en educación superior ha sido un tema recurrente en el campo de la investigación educativa. Incluso se destaca cierto crecimiento en el número de publicaciones en artículos académicos, como se observa en la Tabla 1, donde utilizando como palabras clave: tutoría y educación superior/universidad; mentoring/ tutoring and higher education/university, se realizaron búsquedas en distintas bases de datos considerando tres intervalos de tiempo: 1990-1999; 2000-2009 y 2010-2015.

\section{Tabla 1. Frecuencia de artículos sobre Tutoría en Educación Superior/Universidad en distintas bases de datos}

\begin{tabular}{ccccccc}
\hline & \multicolumn{2}{c}{$\begin{array}{c}\text { Tutoría y Educación Superior/ } \\
\text { Universidad }\end{array}$} & \multicolumn{2}{c}{$\begin{array}{c}\text { Mentoring/Tutoring and Higher } \\
\text { Education/University }\end{array}$} \\
\hline Bases de datos & $1990-1999$ & $2000-2009$ & $2010-2015$ & $1990-1999$ & $2000-2009$ & $2010-2015$ \\
\hline IRESIE & 0 & 28 & 13 & 0 & 0 & 0 \\
\hline REDALYC & 0 & 20 & 21 & 0 & 0 & 1 \\
\hline Dialnet & 38 & 70 & 220 & 0 & 0 & 0 \\
\hline $\begin{array}{c}\text { Academic Search } \\
\text { Complete }\end{array}$ & 0 & 3 & 3 & 14 & 39 & 63 \\
\hline ERIC & 0 & 0 & 0 & 246 & 695 & 575 \\
\hline TOTALES & 38 & 121 & 257 & 260 & 734 & 639 \\
\hline
\end{tabular}

Fuente: Elaboración propia.

El análisis de los artículos académicos recuperados de la búsqueda referida, permite apreciar que en el estudio de la tutoría en educación superior se han definido funciones y actividades específicas del tutor (Campos \& Oliveira, 2013; Vales, Ramos \& Olivares, 2009; De Oliveira \& Rego, 2008; Sobrado, 2008); se han abordado desde la visión de tutores y tutorados los significados, expectativas y prácticas tutoriales (Cid \& Pérez, 2006; Lobato, Arbizu \& Del Castillo, 2004; Santiago, 2006; Troyano \& García, 2009); se ha indagado sobre la implementación de la tutoría tanto presencial como en línea (Álvarez, 2008; Cano, 2008; Capelari, 2014; Martínez, Pérez \& Martínez, 2016; Pera, Gisbert \& Isús, 2007); se han analizado las contribuciones de la tutoría a la formación integral de los estudiantes y a una educación de calidad (Álvarez, 2013; Badillo, 2007; Fernández, Arco, López \& Heiborn, 2011; Larose, Cyrenne, Garceau, Harvey, Guay, Godin, Tarabulsy \& Deschênes, 2011); además se han construido y va- 
lidado instrumentos para medir y evaluar el quehacer tutorial (Caldera, Carranza, Jiménez \& Pérez, 2015; Crawford, Randolph \& Yob, 2014; Sáiz-Manzanares, Bol-Arreba \& Payo-Hernanz, 2014). Sin embargo, aún se carece de entramados conceptuales robustos y explícitos que sustenten la acción tutorial. En aras de abonar a la discusión a través de este documento, se pretende analizar la actividad tutorial desde diferentes corrientes o paradigmas psicológicos de uso frecuente en el campo educativo, con el propósito de valorar las implicaciones prácticas que cada uno ofrece al diseño, implementación y evaluación de programas de tutoría en educación superior. Dada la estructura y el alcance del documento, éste se caracteriza como un estudio temático de índole teórica. Los paradigmas que se analizarán son: el psicoanálisis, el conductismo, el cognoscitivismo, el humanismo, el constructivismo psicogenético y el sociocultural. No es intención de quien escribe realizar un análisis exhaustivo de cada paradigma, pues dicha empresa implicaría discutir corrientes y tensiones al interior de cada uno, por lo que el análisis se limitará a valorar grosso modo cada corriente y así trazar posteriormente implicaciones para el quehacer tutorial en cuanto a sus propósitos, rol de tutores y tutorados, actividades y organización, lo cual podría configurar distintos modelos de tutoría basados en referentes teóricos específicos.

\section{Metodología de revisión}

El abordaje que se presenta en las siguientes páginas es producto de un análisis de diferentes corrientes psicológicas y sus posibles aplicaciones al quehacer tutorial en educación superior. Para dicho análisis se siguieron los siguientes pasos:

a. Se seleccionaron corrientes psicológicas de alta relevancia para el campo educativo. Su trascendencia se tasó tanto por sus aportes para comprender fenómenos educativos como por la frecuencia con que se utilizan como marcos teóricos. Las corrientes seleccionadas fueron: el psicoanálisis, el conductismo, el cognoscitivismo, el humanismo, el constructivismo psicogenético y el sociocultural.

b. Previo a la revisión de cada corriente psicológica se plantearon las siguientes preguntas: ¿qué implicaciones para la tutoría en educación superior podrían desprenderse de las corrientes psicológicas seleccionadas?; ¿qué tipo de anclajes teóricos podrían derivarse de cada corriente psicológica para fundamentar la labor de la tutoría en educación superior?; desde cada corriente psicológica 
¿cómo sería posible conceptualizar los propósitos de la tutoría, así como los roles de tutores y tutorados?; ¿cada corriente psicológica podría aportar a configurar modelos diferenciados de tutoría y de qué manera contribuirían a la formación de los estudiantes de educación superior?

c. Para caracterizar a cada corriente se revisaron tanto escritos elaborados por autores clave como materiales de consulta.

d. Se elaboraron síntesis con los componentes básicos de cada corriente psicológica.

e. Atendiendo a la síntesis de cada corriente se procedió a analizar cuáles podrían ser algunas implicaciones para la tutoría en educación superior.

Es importante mencionar que el análisis permitió identificar actividades que sin duda realizan los tutores, sin embargo, el aporte de este trabajo radica en articular dichas actividades con corrientes teóricas, lo cual facilitará clarificar propósitos, planear y desarrollar la labor de la tutoría con anclajes específicos.

A continuación se presentan los resultados del análisis de cada corriente psicológica. Es importante señalar que al concluir el análisis puntual de las seis corrientes psicológicas, el lector podrá revisar de manera holística cuáles son las implicaciones de dichas corrientes a la tutoría en cuanto a propósitos, roles de tutores y tutorados (Tabla 2); además podrá identificar esquemas de organización para la tutoría (Tabla 3 ) congruentes con cada corriente.

\section{Corrientes psicológicas y tutoría}

\subsection{Psicoanálisis y tutoría}

Si bien dentro del psicoanálisis se encuentran corrientes diferenciadas e incluso autores antagónicos, nos centraremos en algunos rasgos pertenecientes a la corriente freudiana. En general, el psicoanálisis puede ser entendido como una teoría sobre el funcionamiento psíquico humano y la personalidad; un método que investiga los aspectos inconscientes de la vida psíquica humana a través de sus manifestaciones en la libre asociación de ideas, en los sueños y fantasías y en los actos fallidos e involuntarios; una forma de terapia que opera mediante el descubrimiento de las raíces inconscientes de las emociones y de los actos de la persona (Nye, 2002). Si bien el psicoanálisis propiamente no es un paradigma educativo sino de orden clínico, es de llamar la aten- 
ción que dada la forma tradicional en que se desarrollan comúnmente las sesiones de tutoría (relaciones bipersonales en espacios acotados como los cubículos de los tutores), con frecuencia se llega a asumir que la función del tutor es similar al quehacer del terapeuta, condición que en cierto sentido ha desvirtuado el propósito de la tutoría en la educación superior, pues pareciera que aquélla se reduce a un espacio donde el estudiante externa sus problemas y conflictos (de distinta índole y complejidad) en tanto el tutor plantea preguntas abiertas para que el estudiante se explaye, escucha y aconseja. Al respecto, un par de precisiones. Si bien la tutoría en educación superior es una estrategia que pretende contribuir al desarrollo y crecimiento de los estudiantes como personas, es importante delimitar las actividades tutoriales al ámbito académico. Cuando cuestiones personales e incluso de salud aquejan el desempeño de los estudiantes, justo en ese momento debe canalizarse a otras instancias y programas, pues de otra suerte se distorsionan roles y responsabilidades. La segunda precisión tiene que ver con que el psicoanálisis ofrece un componente de alta relevancia para la tutoría: la configuración de un yo equilibrado en los estudiantes, entendiendo que el yo representa la parte racional, reflexiva y realista de la personalidad. En un estudiante de educación superior un yo equilibrado le permitirá interactuar de manera efectiva con otros profesionales, al mismo tiempo favorecerá que valore su propio desempeño $y$, en consecuencia, genere estrategias para mejorarlo de manera continuada.

\subsection{Conductismo y tutoría}

Para fines de este documento se analizarán las implicaciones del conductismo en la tutoría en educación superior, considerando el esquema clásico del conductismo operante, y por otra parte, las aproximaciones más actuales de dicho paradigma que han devenido en modelos de automanejo y modificación del comportamiento cognitivoconductual. En estos últimos se asume que el individuo tiene un papel activo y es capaz de auto monitorear y reforzar su propia conducta.

Desde el conductismo operante o instrumental se advierte que el aprendizaje se manifiesta en conductas emitidas en presencia de estímulos específicos para obtener recompensas o, en su caso, evitar castigos. Dichas conductas permiten que los individuos operen en ambientes específicos y pueden llegar a generalizarse a otros ámbitos. Extrapolando este paradigma al campo de la tutoría en educación superior, se advierte lo siguiente: Cuando la tutoría se centra exclusivamente en la supervisión del dominio de conocimientos en pro del éxito escolar, es posible identificar un 
rasgo del conductismo operante, en tanto se avizora la figura del tutor como aquel que verifica deficiencias y modifica conductas en aras del éxito escolar (recompensa). Otra forma más sofisticada de analizar el conductismo dentro de la operación de los programas de tutoría, en el caso específico de nuestro país, es la manera en que algunas instituciones han vinculado a la tutoría con estímulos económicos y promociones académicas. Por ejemplo, es común que el otorgamiento de becas a los estudiantes se asocie con que éstos cuenten con el respaldo de un tutor. En el caso de los docentes, desarrollar la función de tutoría llega a asociarse con puntajes y estímulos académicos e incluso económicos. En ambos casos la tutoría se concibe como una vía para obtener recompensas. Es frecuente que cuando la tutoría llega a cruzarse con recompensas de dicha naturaleza se enfrasca en una especie de simulación: los tutorados "hacen que tienen tutor" y los tutores "hacen que tienen tutorados" limitándose en los casos más extremos a firmar informes cada cierto tiempo. A pesar de esto, hay aspectos del conductismo operante que pueden ser de gran utilidad para el quehacer tutorial, en especial aquellos relacionados con el llamado aprendizaje de dominio. En este sentido, los tutores podrían identificar las habilidades y conocimientos de los estudiantes (línea base) y planear intervenciones que incluyan acciones y tiempos específicos orientados al alcance de ciertos dominios del aprendizaje, para lo cual se desintegra el aprendizaje en objetivos específicos. Desde esta perspectiva, los tutores realizarían un seguimiento de los resultados y modificarían la intervención en caso necesario, reforzando los progresos y fomentando la práctica positiva (reemplazando unas conductas por otras).

Las aproximaciones más actuales del conductismo ofrecen pautas relevantes para el quehacer tutorial donde las recompensas dejan de ser exclusivamente externas y se promueve un mayor autocontrol de la persona. Por un lado se ubica el modelo de automanejo, donde para modificar la conducta es la persona quien establece metas, monitorea, evalúa su propio progreso y auto refuerza su comportamiento. Este modelo ofrece pautas relevantes para el quehacer tutorial en tanto los tutores podrían guiar a los estudiantes en el establecimiento de metas académicas y personales, y facilitar estrategias para que éstos sean monitores de su propio progreso de tal suerte que sean capaces de autoevaluarse y auto reforzar conductas asociadas a logros. Por otra parte, el modelo cognitivo-conductual acentúa la importancia de un discurso interno aparejado a cambios conductuales, lo cual implica que la persona es capaz de argumentar el qué, para qué y cómo puede realizar mejoras en comportamientos específicos. Si bien el modelo cognitivo-conductual ha mostrado resultados trascendentes en el campo 
clínico y neuropsicológico en el tratamiento de fobias y adicciones (Camilli \& Rodríguez, 2008; Dávila, 2014; Orgilés, Méndez, Rosa \& Inglés, 2003), se advierte que en el campo de la formación profesional, vía la guía de los tutores, puede emplearse como un modelo para la mejora del aprendizaje, el desarrollo de habilidades intrapersonales (por ejemplo, seguridad, confianza, estabilidad emocional) e interpersonales (por ejemplo, comunicación, trabajo en equipo, negociación).

\subsection{Cognoscitivismo y tutoría}

El cognoscitivismo, paradigma que inicialmente recuperó como analogía el funcionamiento de las computadoras (actualmente en desuso) para representar la organización y el funcionamiento de la mente humana, subraya la importancia de los procesos cognitivos y el estudio de las estructuras mentales en funciones psicológicas (Pozo, 2006), tales como la memoria, la atención, el pensamiento, el lenguaje, entre otros. El cognoscitivismo aplicado al campo educativo ha sido utilizado en el diseño de estrategias y técnicas que facilitan la codificación y organización del conocimiento, así como el fomento de la memoria a largo plazo. Este paradigma ofrece herramientas útiles a la tutoría que pueden traducirse en una gama amplia de técnicas y estrategias que faciliten el aprendizaje de los estudiantes, más aún cuando la formación profesional demanda la configuración de nodos conceptuales que integren el corpus teórico y metodológico del área. En este sentido, los tutores podrían fortalecer la memoria a largo plazo de los estudiantes coadyuvando a que estos estructuren los saberes del área profesional específica mediante estrategias (mnemotecnias, mapas mentales, analogías) para organizar, relacionar y jerarquizar el conocimiento, e incluso fomentar que los estudiantes establezcan nexos con saberes de otros campos profesionales y con ello promover aprendizajes inter y transdisciplinares. Así, los tutores podrían orientar su labor hacia modelar estrategias eficaces para el aprendizaje continuo y guiar a los estudiantes hacia el dominio de conocimientos más precisos y completos. Al respecto habría que destacar que en la actual sociedad del conocimiento, sin bien es importante la configuración de nodos conceptuales sólidos para generar conocimiento e intervenciones profesionales eficaces, la habilidad para reconfigurar dichos nodos (con nuevos conocimientos e incluso divergentes) y contender con la incertidumbre es imprescindible para la formación y el desempeño profesional. Lo anterior es un área de intervención relevante para la tutoría en la educación superior, pues demanda la guía y el apoyo de los tutores hacia la formación de los estudiantes como 
organizadores, reorganizadores y gestores de conocimiento e incluso fomentando que asuman el rol de prosumidores (consumidores y productores de conocimiento), lo cual promueve una cultura de mayor participación y fortalece los valores democráticos en la formación de la ciudadanía (Sánchez \& Contreras, 2012) desde los espacios universitarios.

\subsection{Psicología humanista y tutoría}

La psicología humanista coloca en el centro la tendencia a crecer de las personas. Como representante clave de dicha perspectiva se ubica a Carl Rogers, quien elaboró lo que se conoce como teoría centrada en la persona (Rogers, 1996). Esta teoría se basa en dos grandes supuestos: la tendencia formativa y la tendencia realizadora. El primer supuesto se refiere al crecimiento y evolución de formas simples a configuraciones más complejas. El segundo asume una inclinación natural por desarrollar nuestro máximo potencial. Rogers reconoció estos dos supuestos no sólo en las personas sino en todos los seres vivos y los describió como condiciones sine qua non de la existencia. Por otra parte, la teoría centrada en la persona empata con métodos fenomenológicos en tanto acentúa la importancia de indagar las experiencias conscientes y las percepciones del individuo para determinar la realidad; por ello el conocimiento que se tenga de aquéllas es una vía para comprender la conducta humana. Para Rogers, los dos supuestos de su teoría se ven debilitados por presiones sociales y ambientes negativos que limitan la libertad de las personas. Para contrarrestar dichos efectos, Rogers, desde la práctica terapéutica, propuso tres condiciones: congruencia del terapeuta (mostrarse tal como se es, expresar lo que se siente y se piensa); valoración positiva incondicional (actitud amable, de aceptación y aprecio por el otro, sin juicios ni reservas); empatía (capacidad de percibir y comprender los sentimientos de otra persona desde su perspectiva). Desde el punto de vista de Rogers dichas condiciones contribuyen a la autorrealización y a un funcionamiento más pleno de las personas. Si bien tales condiciones de naturaleza interpersonal caracterizan el trabajo terapéutico centrado en la persona, pueden extrapolarse a cualquier tipo de interacción enmarcada en distintos ámbitos e instituciones sociales (familia, escuela, campo laboral, entre otros). Los aportes de la psicología humanista al campo educativo se han orientado a guiar la labor de los docentes y la interacción con y entre los estudiantes, e incluso ha marcado pautas para el abordaje didáctico de contenidos escolares (por ejemplo, aprender historia a través de generar empatía con personajes icónicos). 
La teoría centrada en la persona ofrece a la tutoría en educación superior pautas relevantes para la dinámica intra e interpersonal entre tutores y estudiantes $y$, por otra parte, ofrece herramientas para propiciar la autorrealización de éstas a fin de que alcancen sus metas personales y profesionales. Sobre los aportes a la dinámica intrapersonal, la teoría centrada en la persona permite reconfigurar el rol de los tutores como personas capaces de mostrarse tal como son y con ello denotar su propia condición humana, lo cual, en potencia, pudiese favorecer interacciones más cercanas con los estudiantes, propiciando relaciones de naturaleza horizontal sin que con ello se difumine el respeto y la responsabilidad como agentes educativos. En el plano interpersonal, la teoría en cuestión ofrece pautas específicas como la valoración positiva incondicional y la empatía hacia los estudiantes, las cuales pueden contribuir a mejorar los lazos afectivos y la comunicación asertiva. Considerar la autorrealización de los estudiantes como parte de la formación profesional permitiría que los tutores centren su atención en identificar cuáles son las metas personales y profesionales de aquéllos (o en su caso, favorecer que establezcan y ponderen metas) y coadyuvar con apoyos de diversa índole (por ejemplo, ofrecer información sobre becas, cursos extracurriculares, intercambios estudiantiles, convocatorias y concursos) para el alcance de dichas metas.

\subsection{Constructivismo psicogenético y tutoría}

Llaman la atención las múltiples corrientes en psicología que pueden identificarse como constructivistas. Así, por ejemplo, Hernández (2008), después de realizar una revisión exhaustiva del campo, identificó cinco grandes corrientes del constructivismo, a saber: psicogenética piagetiana, cognitiva, sociocultural, radical y construccionismo social. Sin embargo, según Hernández (2008, p. 41), dichas corrientes poseen aspectos en común, por ejemplo:

a. Una explicación epistemológica sobre cómo se genera y se transforma el conocimiento, donde se enfatiza el papel activo del sujeto y del propio objeto del conocimiento (como realidad cambiante y dinámica).

b. Intentan superar las discusiones entre empiristas e innatistas.

c. El conocimiento no es una copia de la realidad sino una construcción, lo cual implica una perspectiva relativista sobre la realidad que se describe.

d. Enfatiza el papel del sujeto cognoscente como constructor, re-constructor o co-constructor de representaciones sobre la realidad. 
e. El conocimiento es altamente dependiente del sujeto, de su actividad y del contexto en donde éste se genera.

Sin obviar la amplitud de corrientes constructivistas, en este apartado se describe la denominada psicogenética piagetiana y posteriormente se analizan sus aportes a la tutoría en educación superior. Este tipo de constructivismo prioriza la indagación de la construcción del conocimiento científico y con ello dar cuenta de las bases con las que opera el pensamiento. Para Piaget (1991) las estructuras del pensamiento están integradas por esquemas que nos permiten operar en la realidad. Dichos esquemas en los primeros años están ligados a objetos específicos y concretos hasta alcanzar esquemas de mayor complejidad y abstracción, permitiendo trazar distintas etapas en el desarrollo humano, lo cual ha generado discusiones de fondo sobre la relación entre desarrollo y aprendizaje. Para esta corriente los esquemas de manera continua sufren desequilibrios, en especial por la confrontación con nuevos o diferentes objetos de conocimiento o por lo que se conoce como conflictos cognitivos (ideas contrapuestas o contradictorias). Dichos desequilibrios propician que el individuo, en un continuo proceso dialéctico a través de la asimilación (incorporación de nuevas ideas a los esquemas existentes) y la acomodación (ampliación o cambio de esquemas para acomodar nuevos objetos o eventos) intente establecer equilibrios para operar en el mundo, lo cual permite desarrollar, consolidar y reorganizar estructuras cognitivas cada vez más complejas.

El constructivismo psicogenético ofrece a la tutoría en educación superior aportes que coadyuvan al aprendizaje de los tutorados. Desde esta corriente la tutoría podría tener como centro el diálogo con los estudiantes a fin de desafiar su pensamiento a través de la generación de conflictos cognitivos (donde se tensen o confronten creencias o concepciones intuitivas con los saberes propios del campo profesional) y el planteamiento de retos o preguntas relevantes que trastoquen y movilicen sus estructuras cognitivas y les permitan organizar y reestructurar el conocimiento. Los tutores podrían caracterizarse como agentes que retan y confrontan a los estudiantes con preguntas y problemas por resolver y coadyuvan a que aquéllos construyan aproximaciones (esquemas) más cercanos a los conocimientos propios de la profesión o la disciplina. Por su parte, los tutorados podrían definirse como individuos activos que en una espiral constante modifican sus esquemas previos al confrontarse con problemas y preguntas que les permiten mayor dominio de saberes propios del área. 


\subsection{Constructivismo sociocultural y tutoría}

El constructivismo sociocultural recupera los aportes de la obra de Vygotsky. Resalta el papel de factores sociohistóricos y culturales en la génesis de las funciones psicológicas y en el desarrollo cognitivo de los individuos (Wertsch, 1988). Dichas funciones pueden trazarse a partir de las interacciones con los demás (nivel interpsicológico) y después se internalizan (nivel intrapsicológico). En otras palabras, primero se presentan y modelan a nivel social y después son incorporadas y apropiadas de manera individual. Desde esta corriente la participación activa en prácticas sociales y culturales valoradas por la comunidad, así como los intercambios comunicativos y la mediación de otros, coadyuvan al aprendizaje y a la incorporación plena de los individuos al grupo o comunidad específica. El lenguaje juega un papel imprescindible en tanto le permite al individuo tener acceso a la cultura y, al mismo tiempo, desarrollar de manera paulatina funciones psicológicas cada vez más complejas.

Desde el constructivismo sociocultural, se destacan tres conceptos importantes y de múltiples aplicaciones al campo educativo: Zona de Desarrollo Real (zDR); Zona de Desarrollo Próximo (ZDP) y andamiaje. Dichos conceptos permiten focalizar la atención en el nivel que poseen los estudiantes/aprendices (ZDR) y a dónde podrían llegar si reciben apoyos y ayudas específicas (ZDP); dichos apoyos (andamiajes), para ser adecuados, deben ajustarse al nivel y necesidades de los estudiantes/aprendices, y retirarse paulatinamente hasta que éstos se puedan desempeñar sin ayuda, lo cual permite trazar nuevos ciclos de aprendizaje.

El constructivismo sociocultural ofrece pautas de alta relevancia para la tutoría en educación superior, tanto para su organización como en la definición de actividades. En cuanto a su organización, esta corriente, comparada con las revisadas en las páginas previas, da cabida a la generación de comunidades de aprendizaje integradas por diversos tutores y estudiantes, en tanto se pondera la construcción del conocimiento en ambientes auténticos y de colaboración. Así los procesos de aprendizaje son, ante todo, procesos sociales donde a través de la interacción y el diálogo se comparte y reconstruye una cultura académico-profesional.

En el ámbito de la formación profesional se destacan áreas de trabajo con alto potencial para ser materia de la tutoría, como son el campo de la investigación y la intervención profesional, donde se trasciende el ámbito de las instituciones educativas para abrir espacios a otros contextos y situaciones donde la práctica profesional se desarrolla y transforma. Lo anterior permitiría que gradualmente los estudiantes asu- 
man mayor responsabilidad y control de la práctica profesional. Al analizar el rol de los tutores desde esta corriente se advierte que éstos pueden actuar como mediadores y co-aprendices. Mediadores, porque podrían facilitar andamios y apoyos a los estudiantes para que gradualmente logren mayor dominio del campo profesional (transitando de la ZDR a la ZDP) y se incorporen como agentes activos y legítimos dentro de la propia comunidad profesional. Co-aprendices, porque podrían construir junto con sus estudiantes distintas interpretaciones de los conocimientos que integran el corpus teórico y metodológico de la profesión e incluso configurar aportes originales al campo.

En la Tabla 2 se resumen las posibles aplicaciones de los diferentes paradigmas analizados al quehacer tutorial en educación superior.

Como se observa, los aportes de los diferentes paradigmas psicológicos analizados a la tutoría en educación superior acentúan áreas relacionadas con el desarrollo personal y profesional de los estudiantes, lo cual contribuiría a su formación integral. Así, por ejemplo, mientras que los aportes del psicoanálisis y la psicología humanista contribuyen al desarrollo de un yo equilibrado y al alcance de metas personales y profesionales (autorrealización), algunos aportes del conductismo, el cognoscitivismo y el constructivismo psicogenético pudiesen coadyuvar al desarrollo de estrategias eficaces para el aprendizaje continuo; por su parte, del constructivismo sociocultural se recupera el énfasis en la generación de condiciones para que los estudiantes participen de una manera activa en prácticas sociales y culturales propias de la labor profesional, a través de las cuales puedan incorporarse como agentes sociales que reconfiguran y deconstruyen los saberes propios de la profesión.

Los diferentes propósitos de la tutoría anclados a paradigmas psicológicos (Tabla 2) permitirían, por ejemplo: reconfigurar los estilos de organización de la tutoría; optimizar los programas y servicios con los que cuentan las instituciones de educación superior; definir metas de los programas de tutoría así como mecanismos de evaluación. Así, por ejemplo, en la Tabla 3 se aprecian algunos estilos de organización de la tutoría alineados a la naturaleza de cada paradigma analizado. 
Tabla 2. Aplicaciones de diferentes paradigmas psicológicos a la tutoría en educación superior

\begin{tabular}{|c|c|c|c|}
\hline & $\begin{array}{l}\text { Propósito de la } \\
\text { tutoría }\end{array}$ & Rol del tutor & Rol del tutorado \\
\hline Psicoanálisis & $\begin{array}{l}\text { Coadyuvar a la } \\
\text { configuración de un } \\
\text { yo equilibrado en los } \\
\text { tutorados. }\end{array}$ & Aconseja y canaliza. & $\begin{array}{l}\text { Identifica aquellos } \\
\text { aspectos que le } \\
\text { permiten ser un } \\
\text { mejor estudiante. }\end{array}$ \\
\hline Conductismo & $\begin{array}{l}\text { Reforzar progresos y } \\
\text { fomentar la práctica } \\
\text { positiva (reemplazar } \\
\text { conductas por otras). }\end{array}$ & $\begin{array}{l}\text { Monitorea, refuerza y ajusta } \\
\text { conductas que contribuyan } \\
\text { al aprendizaje de los } \\
\text { tutorados. }\end{array}$ & $\begin{array}{l}\text { Automonitorea y } \\
\text { refuerza su propia } \\
\text { conducta. }\end{array}$ \\
\hline Cognoscitivismo & $\begin{array}{l}\text { Proporcionar técnicas } \\
\text { y estrategias que } \\
\text { faciliten la codificación } \\
\text { y organización } \\
\text { del conocimiento } \\
\text { profesional/disciplinar. }\end{array}$ & $\begin{array}{l}\text { Modela técnicas y estrategias } \\
\text { eficaces para codificar y } \\
\text { organizar el conocimiento; } \\
\text { guía a los tutorados hacia el } \\
\text { dominio de conocimientos } \\
\text { más precisos y completos. }\end{array}$ & $\begin{array}{l}\text { Organiza y produce } \\
\text { información y } \\
\text { conocimiento. }\end{array}$ \\
\hline $\begin{array}{l}\text { Psicología } \\
\text { humanista }\end{array}$ & $\begin{array}{l}\text { Favorecer la } \\
\text { autorrealización de los } \\
\text { estudiantes. }\end{array}$ & $\begin{array}{l}\text { Contribuye al alcance } \\
\text { de las metas personales } \\
\text { y profesionales de los } \\
\text { tutorados. }\end{array}$ & $\begin{array}{l}\text { Pondera metas } \\
\text { personales y } \\
\text { profesionales y traza } \\
\text { rutas para alcanzarlas. }\end{array}$ \\
\hline $\begin{array}{l}\text { Constructivismo } \\
\text { psicogenético }\end{array}$ & $\begin{array}{l}\text { Generar conflictos } \\
\text { cognitivos a fin } \\
\text { de organizar y } \\
\text { reestructurar el } \\
\text { conocimiento } \\
\text { profesional/disciplinar. }\end{array}$ & $\begin{array}{l}\text { Plantea retos y confronta a } \\
\text { los tutorados a través del } \\
\text { diálogo y la solución de } \\
\text { problemas. }\end{array}$ & $\begin{array}{l}\text { Reestructura } \\
\text { esquemas sobre } \\
\text { conocimientos } \\
\text { profesionales/ } \\
\text { disciplinares. }\end{array}$ \\
\hline $\begin{array}{l}\text { Constructivismo } \\
\text { sociocultural }\end{array}$ & $\begin{array}{l}\text { Incorporar de una } \\
\text { manera plena } \\
\text { y legítima a los } \\
\text { estudiantes al } \\
\text { quehacer profesional. }\end{array}$ & $\begin{array}{l}\text { Ofrece andamios y ayuda } \\
\text { ajustada al aprendizaje } \\
\text { de los tutorados; genera } \\
\text { condiciones para que } \\
\text { los tutorados participen } \\
\text { en prácticas sociales y } \\
\text { culturales propias de la labor } \\
\text { profesional, como pudiesen } \\
\text { ser proyectos de intervención } \\
\text { o de investigación. }\end{array}$ & $\begin{array}{l}\text { Participa en prácticas } \\
\text { del quehacer } \\
\text { profesional a través } \\
\text { de la actividad y el } \\
\text { diálogo con pares } \\
\text { y expertos. Dota } \\
\text { de significado y de } \\
\text { relevancia social a la } \\
\text { profesión. }\end{array}$ \\
\hline
\end{tabular}

Fuente: Elaboración propia. 
Tabla 3. Organización de la tutoría a partir de diferentes paradigmas psicológicos

\begin{tabular}{ccc}
\hline $\begin{array}{c}\text { Paradigma } \\
\text { psicológico }\end{array}$ & $\begin{array}{c}\text { Propósito de la } \\
\text { tutoría }\end{array}$ & $\begin{array}{c}\text { Organización sugerida de la tutoría y } \\
\text { representación gráfica }\end{array}$ \\
\hline Psicoanálisis & $\begin{array}{l}\text { Coadyuvar a la } \\
\text { configuración de un } \\
\text { yo equilibrado en los } \\
\text { tutorados. }\end{array}$ & $\begin{array}{l}\text { Bipersonal (tutor-tutorado) con apoyo de programas/ } \\
\text { servicios institucionales. }\end{array}$ \\
\hline
\end{tabular}


Tutoría en Educación Superior: análisis desde diferentes corrientes psicológicas e implicaciones prácticas

Gabriela de la Cruz Flores

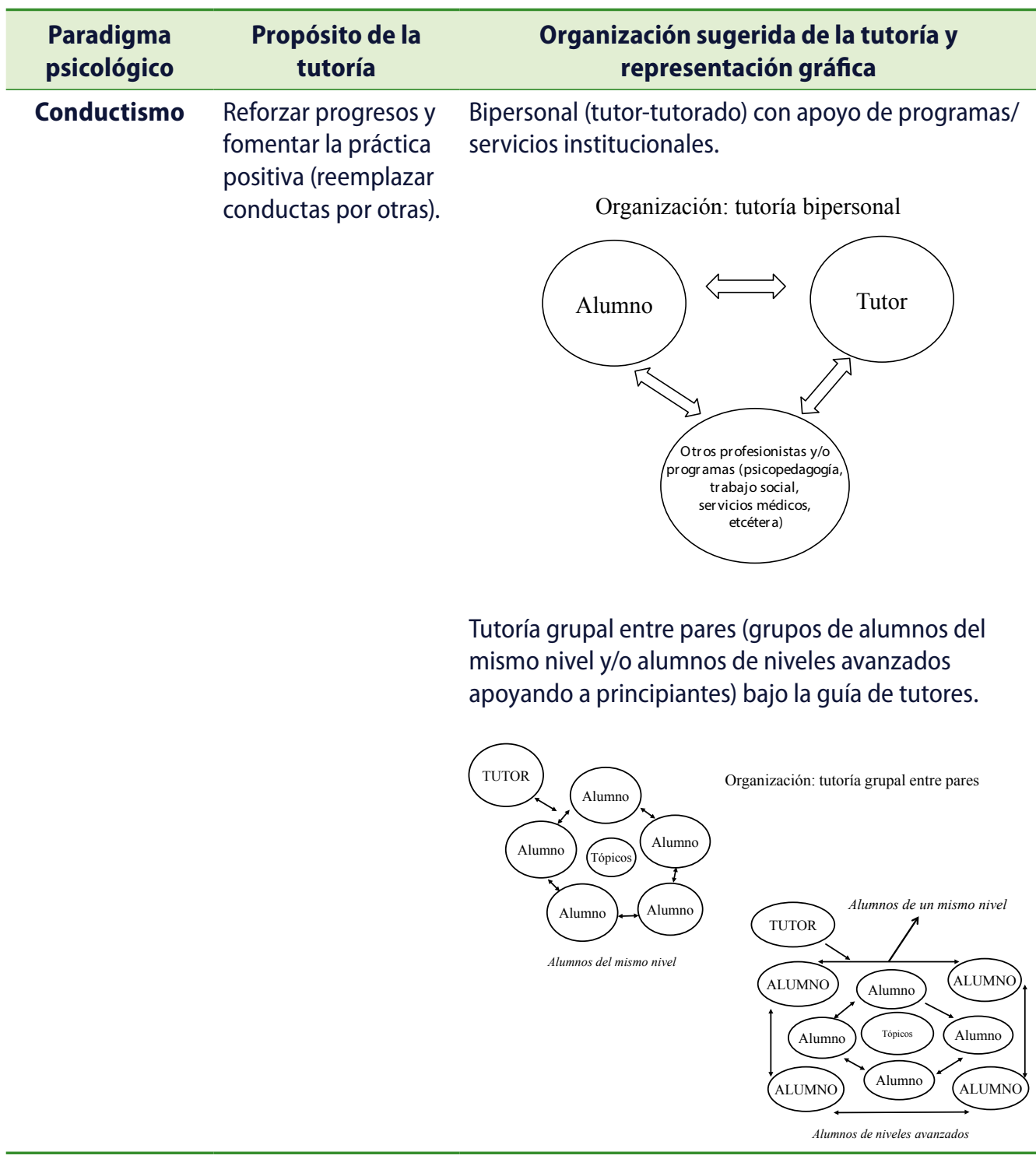


Tutoría en Educación Superior: análisis desde diferentes corrientes psicológicas e implicaciones prácticas

Gabriela de la Cruz Flores

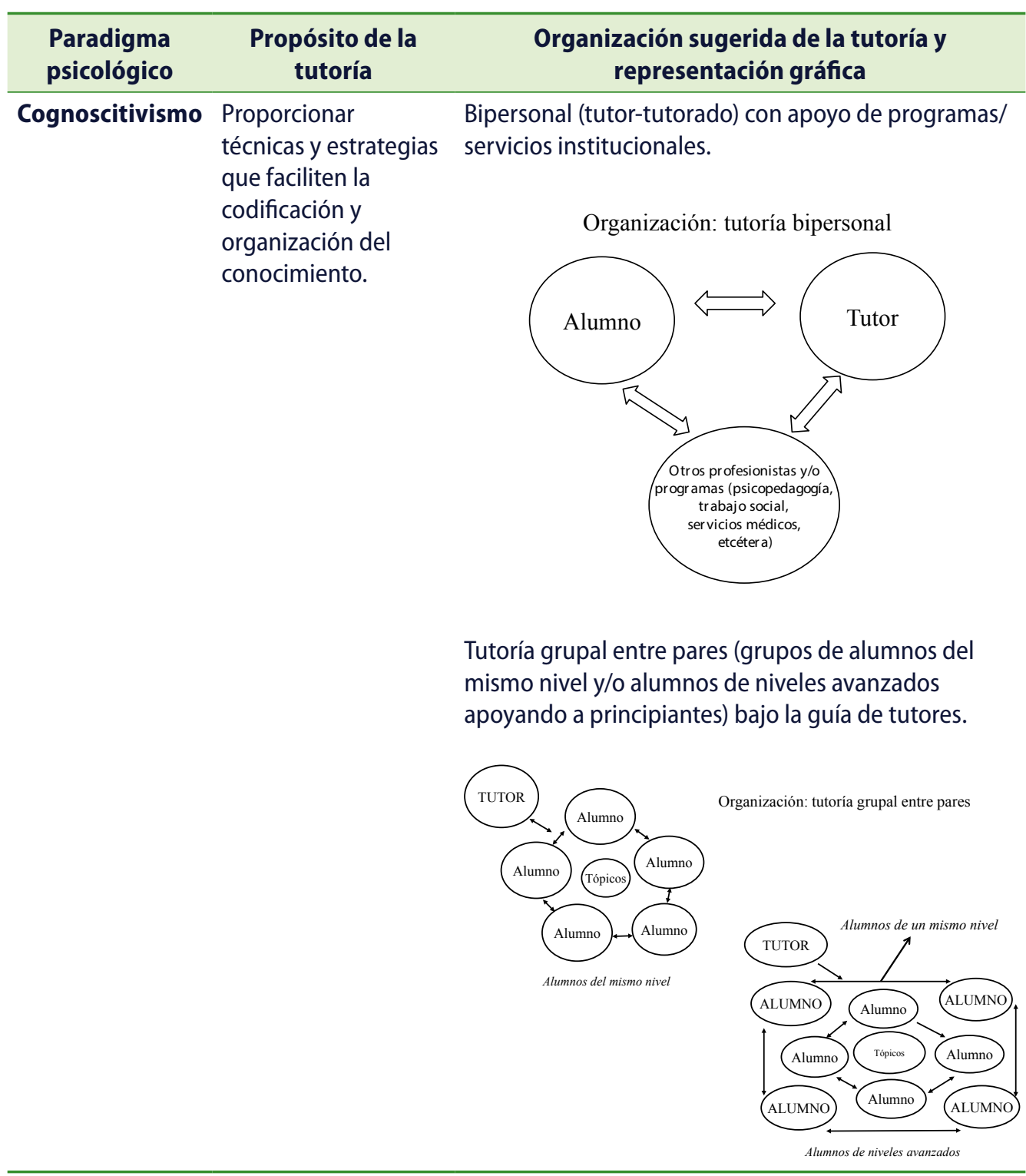


Tutoría en Educación Superior: análisis desde diferentes corrientes psicológicas e implicaciones prácticas

Gabriela de la Cruz Flores

\begin{tabular}{ccc}
$\begin{array}{c}\text { Paradigma } \\
\text { psicológico }\end{array}$ & $\begin{array}{c}\text { Propósito de la } \\
\text { tutoría }\end{array}$ & $\begin{array}{c}\text { Organización sugerida de la tutoría y } \\
\text { representación gráfica }\end{array}$ \\
\hline $\begin{array}{c}\text { Psicología } \\
\text { humanista }\end{array}$ & $\begin{array}{l}\text { Favorecer la } \\
\text { autorrealización de } \\
\text { los estudiantes. }\end{array}$ & $\begin{array}{c}\text { Bipersonal (tutor-tutorado) con apoyo de programas/ } \\
\text { servicios institucionales. }\end{array}$ \\
\hline
\end{tabular}




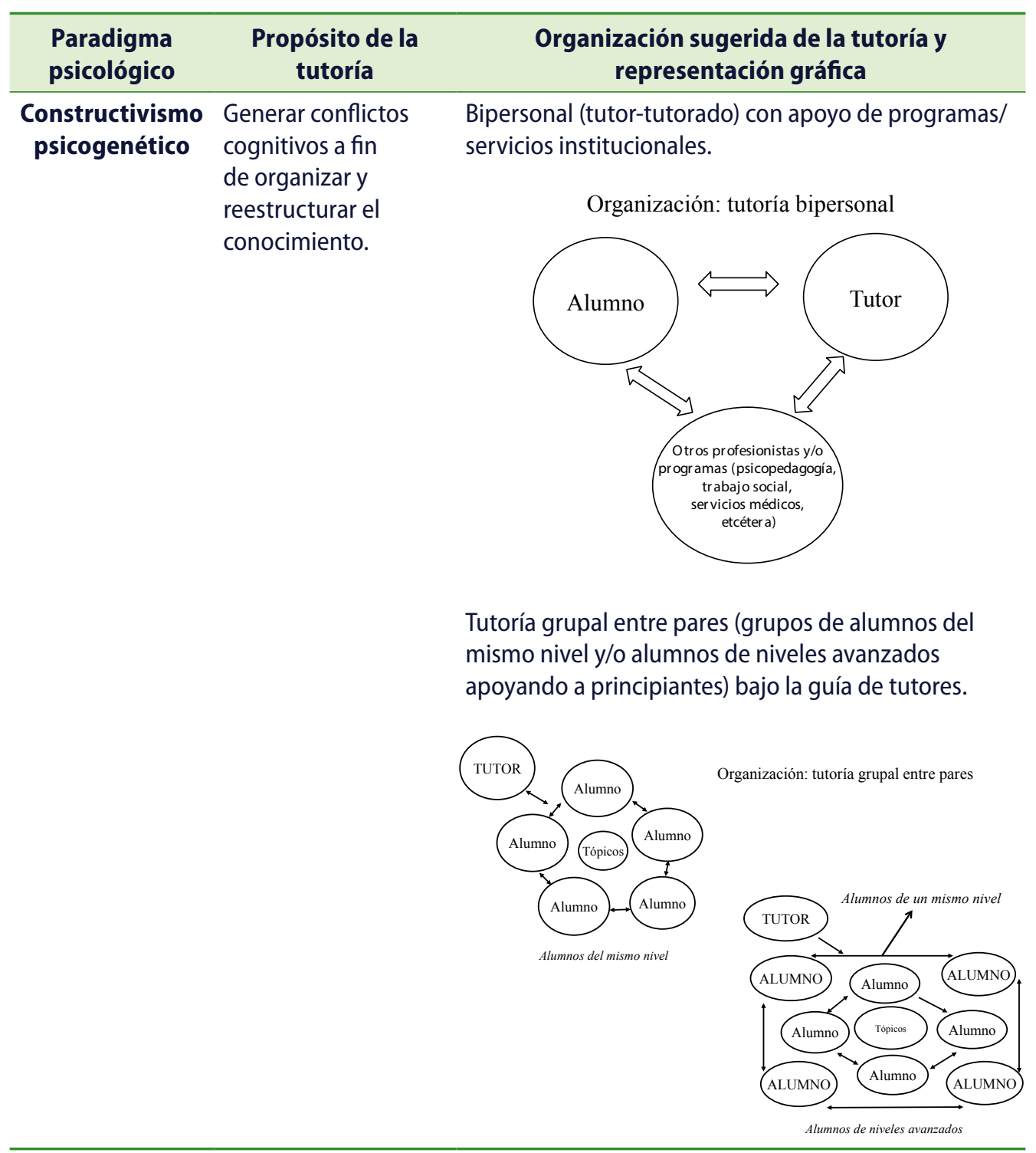




\begin{tabular}{cll}
$\begin{array}{c}\text { Paradigma } \\
\text { psicológico }\end{array}$ & $\begin{array}{c}\text { Propósito de la } \\
\text { tutoría }\end{array}$ & $\begin{array}{c}\text { Organización sugerida de la tutoría y } \\
\text { representación gráfica }\end{array}$ \\
\hline $\begin{array}{l}\text { Constructivismo } \\
\text { sociocultural }\end{array}$ & $\begin{array}{l}\text { Incorporar de una } \\
\text { manera plena } \\
\text { y legítima a los } \\
\text { estudiantes al } \\
\text { quehacer profesional. }\end{array}$ & $\begin{array}{l}\text { Multitutoría: grupos de tutores y tutorados en torno a } \\
\text { problemas/proyectos de investigación/intervención } \\
\text { en escenarios auténticos y relevantes de la práctica } \\
\text { profesional. Los estudiantes gradualmente transitan } \\
\text { de posiciones periféricas a centrales en los grupos de } \\
\text { trabajo. }\end{array}$
\end{tabular}

Fuente: Elaboración propia.

\section{Consideraciones finales}

El análisis expuesto sobre las posibles aplicaciones de diferentes corrientes psicológicas a la tutoría pretende contribuir a la construcción de modelos y estrategias que favorezcan tanto el desempeño de los tutores como la formación de los estudiantes. De esta forma, por ejemplo, los aportes del psicoanálisis y la psicología humanista podrían contribuir al desarrollo de un yo equilibrado y al alcance de metas personales y profesionales, generando modelos de tutoría orientados al desarrollo personal y profesional. Por su parte, algunos aportes del conductismo, el cognoscitivismo y el constructivismo psicogenético podrían utilizarse como anclajes para delinear modelos de tutoría centrados en el perfeccionamiento de estrategias para el aprendizaje continuo. Por último, algunos elementos del constructivismo sociocultural podrían articularse con modelos de tutoría en educación superior, donde se recuperara el énfasis por la generación de condiciones para que los estudiantes participen de una manera activa 
en prácticas sociales y culturales propias de la labor profesional. Cabe hacer mención que este último modelo de tutoría podría ser muy recomendable para incorporar a los estudiantes tanto a prácticas profesionales como al servicio social. En síntesis, cada corriente psicológica puede aportar a la construcción de distintos modelos de tutoría $y$, en su conjunto, contribuir a la formación integral del estudiantado así como a la diversificación de apoyos, dependiendo de sus necesidades formativas y de la temporalidad de los procesos educativos. En este sentido, la tutoría como estrategia de intervención aporta al aprendizaje de cada estudiante, por ello puede concebirse como un mecanismo que favorece la equidad educativa orientada a la mejora de los procesos de aprendizaje. Cabe señalar que este documento pretende aportar al corpus teórico de la tutoría en educación superior, queda pendiente su implementación y posibles ajustes.

Sin duda la incorporación de los programas de tutoría en las instituciones de educación superior ha reconfigurado el propio quehacer docente y ha permitido focalizar la atención hacia los estudiantes. Es importante señalar que las posibles aplicaciones de los diferentes paradigmas psicológicos al quehacer tutorial, pueden ser recuperadas por cualquier tutor independientemente del campo del conocimiento profesional o disciplinar donde se desarrolle; sin embargo, es necesario generar procesos de formación de los tutores y construir estrategias coherentes y consistentes con referentes específicos como los expuestos. Con este documento se pretende aportar a la discusión teórica y didáctica de la tutoría en la educación superior y apuntalar derroteros que enriquezcan la formación profesional y el desarrollo integral de los estudiantes.

\section{Lista de referencias}

Álvarez, M. (2008). La tutoría académica en el espacio europeo de la Educación Superior. Revista Interuniversitaria de Formación del Profesorado, 22(1), 71-88.

Álvarez, P. (2013). La tutoría como eje articulador del proceso de aprendizaje del alumnado. Qurriculum: Revista de Teoría, Investigación y Práctica Educativa, 26, $73-87$.

Asociación Nacional de Universidades e Instituciones de Educación Superior. (2000). Programas Institucionales de Tutoría. Una propuesta de la ANUIES para su organización y funcionamiento en las instituciones de educación superior (col. Biblioteca de la Educación Superior). México: Autor. 
Badillo, J. (julio-diciembre, 2007). La tutoría como estrategia viable de mejoramiento de la calidad de la educación superior. Reflexiones en torno al curso. CPU-e, Revista de Investigación Educativa, 5. Recuperado de http://revistas.uv.mx/index.php/cpue/article/view/115

Caldera, J. F., Carranza, M. R., Jiménez, A. A., \& Pérez, I. (enero-marzo, 2015). Actitudes de los estudiantes universitarios ante la tutoría. Diseño de una escala de medición. Revista de la Educación Superior, 44(1), 103-124.

Camilli, C. R., \& Rodríguez, A. (2008). Fobia social y terapia cognitivo-conductual: definición, evaluación y tratamiento. Anales de la Universidad Metropolitana, $8(1), 115-137$.

Campos, A. C., \& Oliveira, A. H. (2013). A identidade do professor Tutor. Revista ADAPECI, Educação a Distância e Práticas Educativas Comunicacionais e Interculturais, 13(1), 23-37.

Cano, R. (2008). Modelo orga nizativo para la planificación y desarrollo de la tutoría universitaria en el marco del proceso de convergencia europea en Educación Superior. Revista Interuniversitaria de Formación del Profesorado, 22(1), 185-206.

Capelari, M. (2014). Las políticas de tutoría en la educación superior: génesis, trayectorias e impactos en Argentina y México. Revista Latinoamericana de Educación Comparada, 5(5), 41-54.

Cid, A., \& Pérez, A. (2006). La tutoría en la Universidad de Vigo según la opinión de los profesores. Revista de Investigación Educativa, RIE, 24(2), 395-422.

Crawford, L., Randolph, J., \& Yob, I. M. (2014). Theoretical development, factorial validity, and reliability of the online graduate mentoring scale. Mentoring $\&$ Tutoring: Partnership in Learning, 22(1), 20-37.

Dávila, W. (2014). Terapia cognitivo-conductual en el trastorno obsesivo-compulsivo. Norte de Salud Mental, 12(49), 58-64.

Fernández, F., Arco, J., López, S., \& Heilborn, V. (2011). Prevención del fracaso académico universitario mediante tutoría entre iguales. Revista Latinoamericana de Psicología, 43(1), 59-71.

Hernández, G. (2008). Los constructivismos y sus implicaciones para la educación. Perfiles Educativos, 30(122), 38-77.

Larose, S., Cyrenne, D., Garceau, O., Harvey, M., Guay, F., Godin, F., Tarabulsy, G., \& Deschênes, C. (2011). Academic mentoring and dropout prevention for students in math, science and technology. Mentoring \& Tutoring: Partnership in 
Learning, 19(4), 419-439. http://dx.doi.org/10.1080/13611267.2011.622078

Lobato, C., \& Ilvento, M. (mayo-agosto, 2013). La orientación y tutoría universitaria: una aproximación actual. Revista de Docencia Universitaria, 11(2), 17-25.

Lobato, C., Arbizu, F., \& Del Castillo, L. (2004). Las representaciones de la tutoría universitaria en profesores y estudiantes: estudio de caso. Educación XXı: Revista de la Facultad de Educación, 7, 135-169.

Martínez, P., Pérez, J., \& Martínez, M. (2016). Las Tics y el entorno virtual para la tutoría universitaria. Educación XX1: Revista de la Facultad de Educación, 19(1), 287-310. http://dx.doi.org/10.5944/educxx1.13942

Nye, R. (2002). Tres Psicologías-Perspectivas De Freud, Skinner y Rogers. México: Thomson International.

Oliveira De, S., \& Rego, S. (julio-septiembre, 2008). Preceptor, supervisor, tutor e mentor: quais sao seus papeis. Revista Brasileira de Educação Médica, 32(3), 363-373. http://dx.doi.org/10.1590/So100-55022008000300011

Orgilés, M., Méndez, X., Rosa, A. I., \& Inglés, C. J. (2003). La terapia cognitivo-conductual en problemas de ansiedad generalizada y ansiedad por separación: un análisis de su eficacia. Anales de Psicología, 19(2), 193-204.

Pera, S. M., Gisbert, M., \& Isús, S. (octubre, 2007). E-tutoría: uso de las tecnologías de la información y comunicación para la tutoría académica universitaria. Teoría de la Educación. Educación y Cultura en la Sociedad de la Información, 8(2), 31-54

Piaget, J. (1991). Seis estudios de Psicología. España: Labor.

Pozo, I. (2006). Adquisición del conocimiento. Cuando la carne se hace verbo $\left(2^{\mathrm{a}} \mathrm{ed}\right.$.). España: Morata.

Rogers, C. (1996). El proceso de convertirse en persona. México: Paidós.

Sáiz-Manzanares, M. C., Bol-Arreba, A., \& Payo-Hernanz, R. (2014). Validation of an evaluation tutoring task scale at the university. Electronic Journal of Research in Educational Psychology, 12(34), 835-852.

Sánchez, J., \& Contreras, P. (2012). De cara al prosumidor: producción y consumo empoderando a la ciudadanía 3.o. Icono14, 10(3), 62-84. http://dx.doi.org/10.7195/ ri14.v10i3.210

Santiago, J. A. (septiembre-diciembre, 2006). La tutoría desde las concepciones de los estudiantes de geografía y ciencias de la tierra. Revista Educación y Pedagogía, 18(46), 137-142.

Secretaría de Gobernación. (29 diciembre de 2013). Acuerdo 712 Reglas de Operación del Programa para el Desarrollo Profesional Docente. Diario Oficial de la Fe- 
deración.

Sobrado, L. (2008). Plan de acción tutorial en los centros docentes universitarios: el rol del profesor tutor. Revista Interuniversitaria de Formación del Profesorado, $1(61), 89-107$.

Troyano, Y., \& García, A. J. (junio, 2009). Expectativas del alumnado sobre el profesorado tutor en el contexto del espacio europeo de educación superior. REDU: Revista de Docencia Universitaria, 7(2), 1-8.

Vales, J., Ramos, D., \& Olivares, K. (abril, 2009). La función del tutor en ambientes presenciales y no presenciales. Revista Mexicana de Orientación Educativa, 6(16), 16-19.

Wertsch, J. V. (1988). Vygotsky y la formación social de la mente. España: Paidós. 